\title{
UOMO, AZIONE E RELAZIONE NEL PENSIERO GIURIDICO DI ANTONIO PIGLIARU Pier Giuseppe Puggioni
}

\section{Abstract}

This paper aims to present the philosophical thought of Italian scholar Antonio Pigliaru through a critical approach to his works. By delving into the complex relationship between this author and some of the main intellectual movements of the $20^{\text {th }}$ century, I will show that Pigliaru's concern with the problems of law and the state is to be viewed in the light of his humanistic and relational theory.

\section{Keywords}

Humanism; Personalism; Relational theory; Pluralism; Actual idealism.

\section{Il dialogo di Pigliaru con il suo tempo}

A distanza di cinquant'anni dalla sua morte, dopo molti studi condotti intorno suo pensiero, si può dire - in modo solo apparentemente retorico - che il filosofo italiano Antonio Pigliaru (1922-1969) abbia ancora qualcosa da dire. Questo autore è particolarmente noto, per lo meno nel contesto italiano, grazie ad una fortunata ricerca sul fenomeno del banditismo in Sardegna, il cui esito - il saggio su La vendetta barbaricina come ordinamento giuridico (1959) - costituisce un importante contributo non soltanto alla sociologia giuridica, ma anche alla teoria ed alla filosofia del diritto. Tuttavia, l'attenzione del filosofo sardo nei confronti 
delle ragioni teorico-giuridiche della vendetta e del banditismo costituisce solo una parte della sua riflessione, che copre invece molti temi fortemente presenti nel dibattito filosofico-politico novecentesco. Non si può negare, d'altronde, che gli stessi scritti sul banditismo rappresentino il portato del vasto orizzonte speculativo di un intellettuale il quale, nel corso di una vita troppo breve, si è occupato di teoria del diritto e dello Stato, delle nozioni di democrazia e autonomia, della pena, della concezione materialistica della storia e della cosiddetta «lotta per il diritto».

Si deve notare, peraltro, come gli studi sul pensiero pigliariano raramente si siano soffermati sugli aspetti teorici e filosofici che, lungi dal presentarsi secondo un'attenta sistematica, debbano cogliersi attraverso la lettura sinottica di più opere. Un approccio siffatto è fondamentale per comprendere le potenzialità speculative della riflessione politico-giuridica dell'autore, se si considera anche l'ipotesi che la sua morte prematura non gli abbia dato il tempo di raggiungere quella maturità intellettuale necessaria a dotare di ordine e sistematicità le proprie idee. Quello di Pigliaru appare, infatti, un pensiero tormentato, che si esprime secondo forme in continua evoluzione e sente il bisogno costante di appoggiarsi all'insegnamento di altri grandi autori. Ciò nondimeno, nelle sue prospettazioni vi sono molti aspetti di originalità, che si respirano già nelle vecchie pagine di alcuni articoli mai riediti, oltre che nelle opere più importanti e maggiormente studiate. Dalla lettura della produzione pigliariana emerge un'attenzione particolare verso il «problema dell'uomo» ${ }^{1}$, espressione con cui l'autore allude all'indagine sulle condizioni e sui caratteri propri della vita umana. Tali condizioni e caratteri, infatti, servono a riconoscere il soggetto dell'azione nell'uomo in quanto «uomo» (nella misura in cui, cioè, esso presenti alcune caratteristiche che lo individuano epistemologicamente) e si realizzano non soltanto ab externo, ma anche e soprattutto in interiore homine.

1 L'espressione, spesso richiamata negli studi su questo autore, si trova in A. Pigliaru, La piazza e lo Stato (1961), ora in Id., Scritti di Scienza politica, Editrice Dattena, Cagliari 1975, p. 48. 
Attraverso una lettura trasversale delle sue opere, allora, si può constatare come il tenore della riflessione pigliariana, seppur connotato da una notevole variazione di accenti e stili, faccia risuonare lo stesso motivo ad ogni tappa del suo percorso intellettuale. La dimensione dell'umano si pone al centro del suo orizzonte teorico e filosofico, divenendo così il fuoco attorno al quale si muovono le riflessioni sui grandi temi del Secondo dopoguerra e sulle problematiche locali o regionali. Pigliaru, infatti, dedicò gran parte del suo impegno civile ed intellettuale alla questione dell'arretratezza economica e culturale della Sardegna, prendendo sul serio il tema - che oggi si direbbe «sardista» - dell'autonomia politica della Regione. Ebbene, non vi è alcuna esagerazione nell'affermare che il contributo complessivo dell'autore alla filosofia politica e giuridica possa leggersi alla luce di questa attenzione verso l'uomo, nel suo essere e nel suo agire. A riprova di ciò, è bene notare come Pigliaru tenda a prediligere quegli autori che, seppur in modi diversi, riconoscono l'importanza dell'azione umana come fattore di costruzione e trasformazione del mondo. Richiamando le parole di questi autori, egli ne dà una lettura sui generis che, lungi dal rispecchiare alla lettera il pensiero delle sue auctoritates, fa trasparire la peculiare posizione assunta dal filosofo sardo in rapporto al tema della natura e dell'azione umana.

\section{Un dialogo di Pigliaru col nostro tempo}

Non è possibile fornire, in questa sede, un'attenta ricostruzione del pensiero filosofico dell'autore, in modo da analizzarne i risultati e tirare le somme per quanto riguarda il tema prescelto, dovendosi quindi rinviare a studi futuri la possibilità di colmare le inevitabili lacune di questo contributo, che non potrà approfondire, ad esempio, la complessa relazione che lega Pigliaru al contesto intellettuale e politico della Sardegna. Nel corso di quest'analisi, procederemo ad un richiamo sommario, ma sufficiente, ai più importanti aspetti della riflessione pigliariana, così da avere presenti le maggiori linee del suo contributo speculativo e poterci concentrare sul suo sviluppo intorno al tema dell'uomo e della sua azione. Noteremo, peraltro, come in realtà il percorso intellettuale dell'autore sia caratterizzato da forti elementi di continuità, che ci interessano 
per almeno due ragioni: in primo luogo, la ricorrenza di alcuni concetti o concezioni nei vari momenti di questo percorso ci suggerisce quali tematiche il nostro studioso avesse particolarmente a cuore; inoltre, vedremo come il tema in cui Pigliaru si mostra più coerente ed insistente sia dato proprio dalla concezione relazionale della vita pratica (scil., umana), intesa quale fondamento della realtà politica e giuridica. Vari studi condotti sul filosofo di Orune, d'altra parte, hanno evidenziato gli elementi che segnano discontinuità o incoerenze nel pensiero dell'auto$\mathrm{re}^{2}$, oppure hanno analizzato la sua opera, per così dire, a compartimenti stagni, pervenendo ad una visione limitata del suo pensiero ${ }^{3}$. Tuttavia, è importante riconoscere come altri studiosi abbiano invece cercato di accostarsi a questo autore individuando alcuni fili conduttori all'interno della sua produzione filosofica e teorica ${ }^{4}$. Costoro hanno attentamente

2 In questo modo si può leggere, forse, il severo giudizio di D. Corradini, Pigliaru filosofo della società e della politica, in «Quaderni sardi di filosofia e scienze umane», 1979, 4-5-6, pp. 31-68, secondo il quale convivrebbero nel filosofo di Orune attitudini varie ed inconciliabili, che lo avrebbero condotto ad interpretazioni inammissibili (analogamente, si vedano V. Frosini, L’idealismo giuridico italiano, Giuffrè, Milano 1978, p. 26, e R. Treves, Giovanni Gentile, il fascismo e l'ideologia fascista, in «Rivista storica italiana», 1961, pp. 826-831). Come ha giustamente notato Antonio Delogu, sarebbe scorretto tentare di individuare «due Pigliaru, difficilmente componibili: l'attualista che affronta i problemi della morale e della politica (e che eticizza tutto) e l'intellettuale che discute su questioni concrete» (A. Delogu, Linsegnamento di Capograssi nel pensiero di Pigliaru, in Pigliaru, La lezione di Capograssi, Spes, Roma 2000, p. 42), quando in realtà la riflessione pigliariana presenta rimarchevoli aspetti di continuità e di originalità.

3 Tale difetto deve attribuirsi, purtroppo, all'opera di T. Marci, L’idea di persona o la persona come «idea». Il personalismo giuridico di Antonio Pigliaru nel suo contesto culturale, Rubbettino, Roma 2012, che approfondisce per lo più la lettura dei saggi su Persona umana e ordinamento giuridico (1953) e sul Personalismo comunitario (1950).

4 Esemplari, in tal senso, sono gli studi di V. Atripaldi, Il pluralismo come teoria dello Stato: il contributo di Antonio Pigliaru, in Unità dello Stato e pluralità degli ordinamenti, Edizioni Iniziative Culturali, Sassari 1994, e di P. Carta, Pensiero giuridico e riflessione politica in Antonio Pigliaru: dalla lezione di Capograssi all'eredità di Gram$s c i$, in «Quaderni fiorentini per la storia del pensiero giuridico moderno», XXXVII (2008), pp. 349 e ss. 
studiato i saggi pigliariani, cercando le intuizioni più originali ed autentiche dell'autore, all'interno di un marasma fondamentalmente inorganico di citazioni e rinvii.

Il bisogno di segnalare tali studi non si deve tanto ad una, pur doverosa, onestà intellettuale, quanto piuttosto all'intuizione - che li accomuna alla nostra ricerca - secondo la quale cercare di intendere il pensiero di Pigliaru incasellandolo nelle correnti con cui egli, via via, si confronta, è una strategia parzialmente fuorviante. Quella di accostarsi ad un autore attraverso altri pensatori, più celebri e meglio conosciuti, è senza dubbio una scelta utile, dal momento che permette di cogliere il legame che avvince il primo ai secondi, ma per essere euristicamente efficace deve fermarsi dopo la prima lettura. Si correrebbe il rischio, altrimenti, di cercare continuamente il pensiero dell'autore "maggiore" nelle parole dell'autore "minore", rivelandosi infine quest'ultimo poco più che un discepolo del primo. Ora, se una strategia siffatta appare inopportuna in linea generale, lo è a maggior ragione quando si parli di un autore come Pigliaru, il quale sovente si appoggia al pensiero di Gentile, avvicinandosi tuttavia a Capograssi, a Bobbio, a Gramsci e ad altri autori. Se si applicasse il modello interpretativo sopra citato, si dovrebbe dire - come si è detto - che Pigliaru è, di volta in volta, "gentiliano", "capograssiano", "marxista" ecc. Da qui, si potrebbe allora concludere - come si è concluso - nel senso della manifesta incoerenza e inconsistenza della riflessione pigliariana, che tutto sommato non merita di essere «mitizzata $»^{5}$.

$\mathrm{Al}$ contrario, sembra più utile un approccio che, senza disconoscere l'importanza dei modelli e dei grandi autori con cui Pigliaru si confronta, tenti di ravvisare alcune caratteristiche proprie del filosofare pigliariano. Si tratta, in altre parole, di sforzarsi per riconoscere - nei limiti delle possibilità offerte dall'analisi propria delle scienze umane - che alcune affermazioni svolte Pigliaru, spesso riferendosi ad altri autori,

5 Così si desume dalle parole di Corradini, Pigliaru filosofo della società e della politica, cit., p. 32 . 
rappresentano elementi almeno in parte originali della sua riflessione. Com'è stato correttamente notato, egli non si limita ad interpretare - lo vedremo - in modo abbastanza originale il pensiero dei suoi maestri, ma nel rileggerli egli inevitabilmente ripensa se stesso e «parla un po" di sé» ${ }^{6}$. In questa prospettiva, dunque, può svolgersi l'indagine sulla concezione "pigliariana" dell'azione umana nel mondo, per scorgerne l'evoluzione entro il percorso intellettuale dell'autore. Seguiremo, pertanto, lo sviluppo del discorso pigliariano sulle categorie di «uomo» e di «relazione umana», tratteggiando le tappe di tale percorso e analizzando il rapporto del nostro autore con le principali correnti filosofico-politiche e filosofico-giuridiche del suo tempo.

\section{Attualismo e dimensione del noi: un'esperienza teorica e pratica}

Maestro fondamentale di Pigliaru, nel pensiero e nella pratica, fu senz'altro Giovanni Gentile (1875-1944), al cui insegnamento il nostro autore rimase sempre fedele, facendo della pedagogia gentiliana un vero e proprio «stile di vita ${ }^{7}$, impostando su di essa il proprio approccio didattico durante l'insegnamento universitario ${ }^{8}$, come risulta dalle importanti testimonianze dei

6 Cfr. P. Carta, Democrazia e lotta per il diritto, introduzione a A. Pigliaru, Struttura, soprastruttura e lotta per il diritto (1965), Il Maestrale, Nuoro 2012, p. XXVI.

7 Come è noto, infatti, il filosofo francese Pierre Hadot (1922-2010) ha espresso il legame tra pensiero filosofico antico e stile di vita in vari scritti, tra cui ricordiamo P. Hadot, Che cos'è la filosofia antica? (1995), Einaudi, Torino 1998, nonché Id., La filosofia come modo di vivere. Conversazioni con 7. Carlier e A.I. Davidson, Nino Aragno Editore, Torino 2005, dove si afferma che «i filosofi che hanno fondato delle scuole hanno voluto con questo proporre dei modi di vita», in quanto nella formazione del loro pensiero «il fattore principale mi sembra essere la rappresentazione di un certo modo di vivere, quello di un politico illuminato dalle Idee per Platone, di un sapiente contemplatore della Natura per Aristotele, di un saggio beato della sua pace interiore per Epicuro» (ivi, p. 155).

8 Così ricorda, peraltro, M. Puliga, Antonio Pigliaru. Cosa vuol dire essere uomini, Ets - Iniziative Culturali, Sassari 1996, p. 96. L'eredita della pedagogia gentiliana 
corsi seminariali tenuti all'Università di Sassari ${ }^{9}$. D'altra parte, il filosofo siciliano e la dottrina attualistica hanno esercitato una fortissima influenza nel percorso intellettuale del nostro autore, tanto che, ancora negli anni Sessanta, troviamo alcuni importanti richiami alle opere di Gentile ${ }^{10}$. Pertanto, apprestandoci a declinare la riflessione pigliariana sulla costituzione del soggetto e sul rapporto tra soggetto umano e azione, pare opportuno riferirsi in primo luogo all'approccio dell'autore sardo nei riguardi dell'attualismo. In questa fase, infatti, le categorie di cui parliamo cominciano a sviluppare le proprie caratteristiche "embrionali", definendosi nel loro nucleo concettuale proprio in seno al discorso attualista di Pigliaru.

Il rapporto con Gentile è una questione centrale per comprendere l'impalcatura fondamentale della riflessione del nostro autore, posto che tra gli anni Quaranta e Cinquanta si registra in lui una «dipendenza diretta dall'impianto teoretico e dal codice linguistico del pensatore siciliano», che pare riflettersi nella stessa struttura delle sue prime opere $^{11}$. Occorre, tuttavia, chiedersi quale significato il giovane filoso-

in Pigliaru viene attentamente studiata nel saggio di L. Caimi, Motivi pedagogici e impegno educativo in Antonio Pigliaru, Vita e Pensiero, Milano 2000.

9 Merita un cenno l'Introduzione al corso di dottrina dello stato per l'anno 19621963, raccolto successivamente in A. Pigliaru, Scritti di Scienza politica, cit., pp. 83 e ss. Offrendo agli studenti la possibilità di approfondire autonomamente gli argomenti trattati, attraverso una lettura critica che dimostrasse il raggiungimento da parte loro «della persuasione profonda e della massima comprensione» (ivi, p. 88), l'impostazione del corso rivela una concezione del rapporto pedagogico improntata alla massima libertà, «per sottolineare, anche praticamente, l'apertura oggettiva della disciplina; e insieme la complessità delle implicanze morali, politiche, giuridiche di una realtà avvolgente come quella dello Stato» (ivi, p. 87).

10 Basti pensare che, appunto, in Struttura, soprastruttura e lotta per il diritto, cit., p. 41, si cita il Sommario di pedagogia. Vedremo, a tal proposito, come Pigliaru dia una lettura veramente particolare di Gentile, cercando di liberarlo dall'aura di autoritarismo e statalismo da cui per decenni è stato circondato.

11 Così L. Caimi, Motivi pedagogici, cit., p. 34, che evidenzia come negli Appunti di pedagogia (1951), «la messa a fuoco [...] dell'idea stessa di educazione segui[sse] i tipici moduli espositivi del Sommario gentiliano». 
fo sardo attribuisca al vocabolario ed alle categorie gentiliane. Già nel '49, infatti, il nostro autore dà prova di una lettura quanto mai originale del pensiero gentiliano, giungendo ad accostarlo ad alcuni «momenti intermedi» dell'esistenzialismo cristiano ${ }^{12}$, almeno per quanto attiene al rapporto fra conoscenza della verità trascendente ed azione posta in essere per attuare tale verità. In questa sede, Pigliaru comincia a dire qualcosa sul nostro tema, parlando dell'eredità di Gentile come una filosofia «definitivamente riabilitata nel filosofare [...] degli uomini vivi, impegnati a ciò, non per mero esercizio culturale, ma perché meglio ci si possa sentire, nel 'mondo', parte attiva d'una intensa spiritualità riscoperta e affermata senza remore di sorta ${ }^{13}$. Com'è agevole notare, la lettura pigliariana si mostra divergente dall'interpretazione canonica che si dà in relazione al testo di Gentile. Quest'ultimo, infatti, prospetta la sussistenza d'un Io assoluto ed immanente come soggetto del nostro pensiero (tale è l'«atto puro»). L'atto del pensiero ha, per Gentile, carattere universale e non particolare, in quanto il suo soggetto protagonista non è costituito dall'Io empirico (uno tra molti), bensì dall'Uno inteso come Io assoluto. Tale impostazione, dunque, riduce il molteplice all'unità e presenta una serie di implicazioni nel campo della filosofia pratica ed specificamente nel rapporto tra individuo e comunità politica. Il particolare si perde nell'universalità di un «processo spirituale che nel suo seno appiana e supera ogni contrasto d'interessi» ${ }^{14}$, sicché la società viene concepita «in interiore homine» - e non inter homines - nel senso che essa viene a costituire «un uomo solo, che ha un solo interesse, in continuo incremento e svolgimento: il patrimonio dell'umanità» ${ }^{15}$. In Pigliaru, invece, l'Io assoluto, pur non arrivando a coincidere con l'in-

12 A. Pigliaru, Commosso omaggio a Gentile (1949), in Id., Scritti sul fascismo, a cura di M. Addis Saba e M. Puliga, Ets - Iniziative Culturali, Sassari 1983, p. 47.

13 Ivi, p. 48.

14 A. Lo Schiavo, Introduzione a Gentile, Laterza, Roma-Bari 2001, p. 91.

15 G. Gentile, I fondamenti della filosofia del diritto ${ }^{3}$ (1916), Sansoni, Firenze 1961, p. 76. 
dividuo empirico, assume un carattere di profonda concretezza, in virtù dell'enfasi che il nostro autore pone sull'azione. Ora, se l'attualismo esalta il valore dell'azione in termini collettivi ed universali, il filosofo di Orune osserva l'azione in relazione all'uomo vivo e concretamente operante nel mondo, con una serie di importanti conseguenze.

La prima conseguenza è che Pigliaru si avvale delle categorie gentiliane per affrontare alcune questioni concrete - potremmo dire " $\mathrm{di}$ attualità" -, lasciandosi coinvolgere nel dibattito pubblico come intellettuale e filosofo "militante". In questo contesto, ad esempio, egli ha modo di pronunciarsi su di una «dolorosa vicenda giudiziaria», richiamandosi alla pedagogia di Gentile ${ }^{16}$. In secondo luogo, Pigliaru rivede ulteriormente il pensiero del filosofo siciliano, finendo quasi per ribaltarne la lettura classica, portandolo ai limiti della sua stessa «flessibilità concettuale ${ }^{17}$. Negli anni Cinquanta, infatti, il nostro autore pubblica alcuni articoli che non solo confermano la lettura "esistenzialista" dell'attualismo ${ }^{18}$, ma addirittura rendono Gentile un fautore del protagonismo dell'uomo nella società e dell'attribuzione al soggetto, in quanto «uomo» vivo ed operante in società, di varie "prerogative normative" (attributi che devono, cioè, essere riconosciuti, in primo luogo dallo Stato). Per questo Gentile "pigliariano", ricondotto ad una matrice "liberale", il

16 Sul finire degli anni Quaranta, infatti, si pone la questione davanti al giudice civile se, ai fini dell'affidamento del figlio minore, il genitore ateo possa definirsi «buon educatore», categoria giurisprudenziale ricavata dall'art. 155 c.c. Il giovane Pigliaru, richiamando le categorie gentiliane, fa notare come tutto il processo educativo sia caratterizzato da un «intrinseco dinamismo», di fronte al quale «quella particolare teologia d'opinione che è la presunta religiosità d'un genitore o l'ateismo dell'altro (altra religio, se consapevole)» presentano, «in un medesimo giudizio, [...] un'eguale legittimità» (A. Pigliaru, Affidamento della prole, Tipografia Quartara, Torino 1952, p. 17).

17 M. Brigaglia, Antonio Pigliaru, 1922-1969. Note per una biografia, in «Ichnusa», n.s. VIII (1989), 18/19, p. 5.

18 Con questo spirito, infatti, Pigliaru recensisce il libro di V. Bellezza, L'esistenzialismo positivo di Giovanni Gentile, pubblicando un contributo omonimo in «Rivista internazionale di filosofia del diritto», XXXIII (1956), n. 5, pp. 666 e ss. 
valore morale dell'uomo risiede per un verso nella sua libertà, che rappresenta la culla «di quell'assoluta democrazia espressa nella forza del diritto e stabilita per la sua assoluta universalità ${ }^{19}$, e per altro verso nel suo lavoro, poiché lavorando egli si eleva «al regno dello spirito, dove il mondo è quello che egli crea pensando», e si rende «faber sui ipsius» ${ }^{20}$.

Tra le conseguenze dell'originale approccio all'attualismo, la più importante ai nostri fini è l'analisi della relazione umana. Prendendo in esame lo sviluppo dell'io, infatti, Pigliaru scandisce con insistenza il passaggio del soggetto dalla dimensione individuale (io) a quella collettiva (noi). Tale scansione è sì debitrice della lezione gentiliana ${ }^{21}$, ma palesa una tendenza, tutta pigliariana, ad accentuare il momento relazionale entro il ritmo produttivo dell'io. Orbene, svolgendo un lungo discorso sul rapporto tra persona e ordinamento, il nostro autore pone l'accento sul tema della «frattura» e del suo «superamento», due momenti consecutivi nei quali si articola la dinamica relazionale. Ogni relazione umana, infatti, s'inserisce in un «ritmo di produzione» in virtù del quale la persona - nozione che vedremo nel paragrafo seguente - si svolge e si integra nell'ordine che le è proprio, di modo che la relazione ed i suoi passaggi sono tappa imprescindibile della formazione dell'io in quanto

19 A. Pigliaru, Fondazione morale della democrazia nel pensiero di G. Gentile (1953), ora in Id., Scritti di Scienza politica, cit., p. 303.

20 La citazione è tratta da G. Gentile, Genesi e struttura della società. Saggio di filosofia pratica (1943), Sansoni, Firenze 1975, p. 112, sui cui si basa Pigliaru per scrivere Il lavoro e il nuovo umanesimo di Giovanni Gentile, Gallizzi, Sassari, 1953, p. 33.

21 La concezione gentiliana del noi viene affermata con forza nel saggio, già citato, su Genesi e struttura della società, in cui il filosofo siciliano afferma la necessità che gli individui entrino fra loro in relazione e che il soggetto, sperimentando l'incidente dell'alterità, comprenda che quello che gli sta dinanzi è «un alter capace di libertà, ossia di tutta la vita spirituale che il soggetto si trova a possedere», sicché egli «non può non rinunziare alla sua solitudine» (Gentile, Genesi e struttura della società, cit., p. 36). Per superare l' «atomismo sociale e politico» (ivi, p. 13), la teoria gentiliana postula che in fondo all'Io stia un $\mathcal{N}$ oi, «che è la comunità a cui [l'Io] appartiene, e che è la base della sua spirituale esistenza» (ivi, p. 15). 
persona umana. Il primo momento della relazione, la frattura, è dato dall'incontro dell'io con l'altro, nell'ambito del quale il soggetto sperimenta il confronto con un'entità differente, ossia altra da sé dal punto di vista subiettivo. In questo contesto, quel ritmo produttivo della persona sembra arrestarsi, poiché confrontarsi con qualcuno che pare esprimere uno spirito diverso dal mio non dovrebbe, intuitivamente, permettermi di sviluppare in alcun modo la mia personalità. A questo punto, perché il ritmo possa riprendere il proprio corso, deve sopraggiungere il secondo momento della relazione, rappresentato dal superamento della frattura, dato dalla presa di coscienza che, nel profondo, vi sono nell'altro alcuni caratteri che non negano la mia personalità e consentono di instaurare un dialogo sulla base di una certa comunanza di bisogni tra me e lui («gli altri non sono non-Io»). Grazie a questa comunanza, io e l'altro perveniamo ad una vera e propria "comunione", nella quale si rivelano le «ragioni positive della prima frattura» ed in cui si mostra quella omogeneità di fondo in virtù della quale possiamo parlare di un «noi ${ }^{22}$. Dalla sua esperienza attualistica, dunque, Pigliaru trae ispirazione per elaborare la propria categoria del noi, ulteriormente sviluppata nel corso delle altre stagioni della sua produzione, conservandosi intatto questo suo nucleo concettuale.

\section{Il rapporto col pensiero personalista}

Durante la sua esperienza attualistica, Pigliaru si confronta con alcuni autori francesi, che affrontano il tema del soggetto morale, politico e giuridico mettendo in primo piano la categoria di «persona». Tale nozione, peraltro, eserciterà un notevole influsso teorico sulla formazione delle Costituzioni occidentali nel dopoguerra, ponendosi come un simbolo della cesura fra XIX ed XX secolo. La stessa idea di persona espressa

22 A. Pigliaru, Persona umana e ordinamento giuridico (1953), con prefazione di G. Bianco, Il Maestrale, Nuoro 2008, p. 37. 
dalla nuova (come lo era) Costituzione italiana sembra richiamare una visione relazionale del soggetto, in virtù della quale «la formazione della nostra personalità» - afferma Tito Marci - «passa necessariamente attraverso il rapporto con gli altri: con ogni altro raggiungibile anche attraverso i canali dell'istituzione ${ }^{23}$. Tale visione, particolarmente evidente nella formulazione degli articoli 2 e 3, esalta la socialità come carattere proprio dell'umano, che si accompagna al riconoscimento di alcuni doveri reciproci, contrapponendosi alla concezione dell'uomo come «individualità predefinita in termini psicologici e morali», che si limita a parlare il «linguaggio dei diritti» ${ }^{24}$. Vedremo, tra poco, come anche in Pigliaru la nozione di «persona» sia strettamente legata, da un lato, ad un fondamento relazionale e, dall'altro lato, al linguaggio dei doveri.

All'inizio degli anni Cinquanta, vedono la luce alcuni saggi con cui, confermando il proprio gusto per la cultura esistenzialista francese ${ }^{25}$, il nostro autore si avvicina allo studio del personalismo comunitario. Egli cerca, evidentemente, di intercettare il clima intellettuale post-bellico e, con esso, le sue istanze di liberazione integrale dell'uomo ${ }^{26}$, prendendo parte, così, al dibattito sul «problema della persona umana» ${ }^{27}$, conside-

23 Marci, L’idea di persona, cit., p. 25.

24 T. Greco, Il ritorno dei doveri. Una critica della filosofia dei diritti, in S. Mattarelli (a cura di), Il senso della Repubblica. Doveri, FrancoAngeli, Milano 2007, pp. 18-19, dove si dice, riprendendo la nota riflessione del Mazzini (1805-1872), che «solo l'esercizio dei doveri permette di creare un vincolo originario tra gli uomini che non dipendesse - hobbesianamente - dalla forza di qualcuno. Il diritto rimanda all'individualità mentre il dovere implica associazione». Secondo questa concezione, la «filosofia dei doveri» - e non quella dei diritti - rappresenterebbe correttamente il pensiero ispiratore della Costituzione repubblicana.

25 Pigliaru è infatti autore di un Esercizio di lettura su Gabriel Marcel, Gallizzi, Sassari 1949, le cui riflessioni si vengono più volte riprese nella ricerca sul personalismo comunitario.

26 Cfr. Caimi, Motivi pedagogici e impegno educativo, cit., p. 27.

27 A. Pigliaru, Considerazioni critiche su alcune posizioni del personalismo comunitario, Gallizzi, Sassari 1950, p. 3. 
rato un «nodo comune ad ogni esigenza viva della filosofia contemporanea ${ }^{28}$. Orbene, Pigliaru si confronta in prima istanza con il discorso personalista portato avanti Emmanuel Mounier (1905-1950) e dal gruppo orbitante intorno alla rivista «Esprit». Il nostro autore, pur mostrando la propria simpatia verso l'idea mounieriana di una struttura umana «sempre alle prese col contingente $»^{29}$, non nasconde una certa diffidenza nei confronti di quello che ritiene essere un personalismo poco rigoroso nel rispetto dei propri principi. Di tale personalismo «disponibile» Pigliaru denuncia soprattutto il carattere «equivoco», giacché esso gli appare incapace di «saldare interiorità ed esteriorità in un destino unico (il destino dell'uomo nel destino del suo mondo e viceversa)» e di risolvere la perplessità «tra economia e morale ${ }^{30}$. Il filosofo sardo, infatti, rigetta la dicotomia tra volizione del particolare e volizione dell'universale. Per lui, la persona è soggetto che agisce sì nel particolare, ma trascende la propria individualità e, sviluppandosi nella relazione con l'altro, procede tendendo al bene universale. Diversamente, l'individuo in senso proprio, l'homo oeconomicus, si auto-limita permanendo volontariamente nell'agire particolare. Sicché, «l'individualismo come atteggiamento pratico in tanto è tale in quanto rifiuti o respinga quell'arricchimento interiore per cui la persona si fa, nell'individuo, come individualità trascesa, ed aperta senza per altro annullarla allo stesso modo in cui il fine dell'etica non annulla l'economia» ${ }^{31}$.

28 A. Pigliaru, Persona umana e ordinamento giuridico, cit., p. 3.

29 Cfr. A. Pigliaru, Considerazioni critiche, cit., pp. 36-37, dove fa riferimento esplicito al testo di E. Mounier, Révolution personnaliste et communautaire Seuil, $\mathrm{Pa}-$ ris 1935, trad. it. L. Fuà, Rivoluzione personalista e comunitaria, Edizioni di Comunità, Roma 1955.

30 A. Pigliaru, cit., pp. 44-45.

31 Ivi, p. 56. Il filosofo di Orune contesta, infatti, la celebre visione crociana che «riduce» la dimensione giuridica all'agire economico. Sul punto, cfr. B. Croce, Riduzione della filosofia del diritto alla filosofia dell'economia (1907), a cura di C. Nitsch, Giuffrè, Milano 2016; A. Pigliaru, Ciò che è vivo e ciò che è morto nel pensiero di Benedetto Croce, in «Studi sassaresi», (1953), 1. 
Altra importante figura richiamata da Pigliaru è quella di Jacques Maritain (1882-1973), al cui pensiero il nostro autore s'accosta secondo un'interpretazione "manipolatrice" o, per lo meno, adeguatrice rispetto alle proprie idee sul rapporto tra persona e Stato. Così, uno dei padri del personalismo comunitario viene, in un certo senso, ricondotto alla lezione di Gentile, secondo una lettura che tenta di conciliare attualismo e integralismo, al fine di «trovare per l'uno e per l'altro un volto e un fondamento comune», rappresentato dal culto dell'uomo «che dovrebbe (e dovrà) costituire l'umanesimo dei tempi nuovi per il quale e nel cui clima lo Stato stesso in quanto volere come volere comune e universale dovrà essere posto da un'umanità ben aperta ad una eguale volontà universale». Nella visione avanzata da Pigliaru, «lo Stato, in quanto principio d'autotrascendenza, non è altro che un portato diretto della nozione stessa di persona», la quale è per Maritain il «valore più originariamente integrale di tutti i valori umani» ${ }^{32}$. In altri termini, l'autorità politica riceve legittimazione e giustificazione in quanto essa si rivolga al compimento di un «ufficio morale» nel rispetto e nella garanzia della libertà (si parla, infatti di «pedagogia della libertà»), la quale deve a sua volta concepirsi in funzione della categoria pigliariana di «persona». Il nostro autore, infatti, parla di «persona come $i d e a\rangle^{33}$, da intendersi come uno sviluppo «ontologico» ${ }^{34}$ rispetto alla concezione comunitarista «provvisoria» della persona come essere relazionale e sociale. La persona, nel suo esserci in quanto «farsi», non si ferma alla sua singolarità e puntualità, bensì è «morale esercizio di libertà che è morale assunzione di responsabilità ${ }^{35}$ : Pigliaru chiama questo carattere «partecipazione aperta», per alludere al significato normativo del nesso tra libertà e responsabilità che conduce il singolo a percepire il proprio limite

\footnotetext{
32 A. Pigliaru, Considerazioni critiche, cit., p. 76 (corsivi nel testo); p. 93; p. 56.

33 Id., Persona umana e ordinamento giuridico, cit., p. 17.

34 In questi termini si esprime T. Marci, L'idea di persona, cit., p. 68.

35 A. Pigliaru, Persona umana e ordinamento giuridico, cit., p. 57.
} 
nell' «umano rispetto dovuto all'uomo in quanto persona $»^{36}$. Con questo ragionamento, che attraversa la dinamica - di cui abbiamo già parlato del passaggio dall'io al noi, lo studioso sardo ricava «quel supremo criterio deontologico che pone, non improvvisamente ma provvidamente, la persona stessa come norma sui». In definitiva, la persona si realizza, da un lato, prendendo consapevolezza, nella sua libertà, del limite al proprio agire morale, derivante dai doveri all'interno dei rapporti intersoggettivi; dall'altro lato, posto che il concetto di persona implica il principio normativo del dovere, essa si realizza nell'ordine dei rapporti intersoggettivi e nel «diritto» come forma di tale ordine ${ }^{37}$. Non si tratta, tuttavia, di un ordine giuridico che subissa il ruolo del soggetto umano. Certo, per dirla con Sergio Cotta, il diritto funge da «seconda natura» o «garanzia di vita» per l'uomo ${ }^{38}$, ma è su quest'ultimo che si concentra il principio costitutivo del sistema giuridico, essendo egli «attivo soggetto di tutta la complessiva attività giuridica ${ }^{39}$.

Come abbiamo visto, pur essendo la riflessione pigliariana sempre più complessa, l'autore non tralascia un importante ed esplicito riferimento al leitmotiv relazionalista. Il concetto di persona, infatti, richiama il coinvolgimento spirituale del soggetto nel rapporto con l'altro, per cui il legame sociale rappresentato dal no $i$ ha sempre qualcosa in più rispetto al mero rapporto inter-individuale: l'approccio personalista di Pigliaru lo porta a confermare la tesi di matrice gentiliana della società in interiore, risultante dalla partecipazione reciproca tra le singole personalità

$36 \quad$ Ivi, p. 59 [corsivo nel testo]. È interessante notare come questa lettura pigliariana, che individua significativi accenti di relazionalismo politico-giuridico in Maritain e Mounier, in un certo senso anticipi alcuni sviluppi della letteratura critica sul personalismo (si vedano, ad esempio, le conclusioni di P. Viotto, Il pensiero contemporaneo secondo f. Maritain, Città Nuova, Roma 2012, pp. 290-299) ed alcune importanti elaborazioni teoriche degli ultimi decenni (cfr. J.M. Burgos, Introducción al personalismo, Palabra, Madrid 2012; Id, La experiencia integral, Palabra, Madrid 2015).

37 A. Pigliaru, Persona umana e ordinamento giuridico, cit., pp. 59-60.

38 S. Cotta, Soggetto umano e soggetto giuridico, Giuffrè, Milano 1997, pp. 109-112.

39 A. Pigliaru, cit., p. 62. 
«ove l'alterità estrinseca davvero si fa, nel prossimo, alterità intrinseca per un vincolo d'amore più forte d'ogni vicenda ${ }^{40}$. D'altra parte, la nozione di persona come norma sui considera una qualità del soggetto che non può prescindere dalla sfera giuridica. In Pigliaru, infatti, il ritmo produttivo della persona si completa nell'ordinamento giuridico, che statuisce in merito al suo status giuridico ed ai suoi rapporti intersoggettivi. Ora, una prospettiva siffatta cade senza subbio sotto la scure della critica rivolta al personalismo filosofico da Simone Weil (19091943), dove la nozione di «persona» viene completamente decostruita e demistificata, per lo meno nella configurazione datale dalla corrente cui fanno capo anche Maritain e Mounier. Il concetto di «persona», infatti, appare eccessivamente legato ad una necessaria configurazione "giuridica" del soggetto e, dunque, non è in grado di rilevare quel che è veramente «sacro», ossia «tutto ciò che nell'uomo è impersonale» ${ }^{41}$. La soluzione di Pigliaru è, in effetti, ben diversa, giacché egli ritiene il diritto positivo uno strumento di "buono" in quanto è l'unico mezzo atto a garantire la stabilità dei rapporti sociali: nel suo periodo di ferma adesione all'attualismo, d'altronde, egli non può negare all'ordinamento giuridico una funzione costitutiva della persona, in considerazione della quale anche il suo pensiero rientra nella stessa critica mossa nei confronti della corrente francese. Tuttavia, è altresì importante distinguere, sotto un profilo sostanziale (oltre il mero dato nominale), la posizione del filosofo sardo da quella di Maritain o di Mounier. Infatti, la concezione pigliariana della persona si lega fortemente alla dimensione

40 A. Pigliaru, Considerazioni critiche, cit., p. 70.

41 S. Weil, La personne et le sacré, Gallimard, Paris, 1957, tr. it. a cura di M.C. Sala e G. Gaeta, La persona e il sacro, Adelphi, Milano 2012, p. 17. Si veda, in proposito, lo studio di T. Greco, La bilancia e la croce. Diritto e giustizia in Simone Weil, Giappichelli, Torino 2006, p. 122: per la filosofa francese, «l'errore intrinseco al concetto di persona sta non soltanto nel fatto che esso delinea l'individualità dell'uomo attraverso la sfera del proprium [...], ma anche nel fatto che attribuisce all'individuo una perfezione che non gli si addice». 
concreta della vita e dell'azione umana, esaltando, inoltre, il profilo del dovere umano nei confronti dell'altro ed il rapporto di tale dovere con la legittimità del potere. Si tratta, in ambedue i casi, di aspetti profondamente radicati nel comunitarismo weiliano ${ }^{42}$, al quale, allora, potremmo in parte accostare il nostro autore.

\section{La filosofia dell'esperienza giuridica ed il pluralismo}

All'inizio degli anni Cinquanta, l'incontro con Giuseppe Capograssi (1889-1956) segna un significativo mutamento nella prospettiva «metodologico-teoretica» di Pigliaru ${ }^{43}$. L'amicizia tra il giovane studioso sardo ed il «giurista senza aggettivi» ${ }^{44}$ abruzzese rappresenta un importante punto di svolta nel cammino intellettuale del nostro autore, con particolare riguardo alla sua riflessione sul diritto. Lincidenza della lezione capograssiana è così profonda da indurre Pigliaru a rivedere molti punti su cui il suo pensiero sembrava aver trovato un approdo sicuro. Sotto il profilo teoretico, infatti, possiamo registrare uno slittamento del punto focale della filosofia pigliariana, che passa dal ritmo produttivo

42 Cfr. S. Weil, L'enracinement, Gallimard, Paris 1949, tr. it. a cura di L. Boella e F. Fortini, La prima radice. Preludio a una dichiarazione verso l'essere umano, Leonardo, Milano 1996, p. 25, dove l'eguaglianza, «bisogno vitale dell'anima», viene eletta a scopo delle regole e principio fondativo, insieme alla libertà, della legittimità: «essa consiste - afferma la filosofa francese - nel riconoscimento pubblico, generale, effettivo, espresso realmente dalle istituzioni e dai costumi, che a ogni essere umano è dovuta la stessa quantità di rispetto e di riguardo, perché il rispetto è dovuto all'essere umano come tale e non conosce gradi». Sul punto, si veda anche T. Greco, «Distinguere la vera grandezza da quella falsa». Sulla legittimità delle istituzioni in Simone Weil, in R. Fulco, T. Greco (a cura di) L'Europa di Simone Weil. Filosofia e nuove istituzioni, Quodlibet, Macerata 2019, p. 62.

43 In questi termini riflette Antonio Delogu, filosofo morale e attento studioso del pensiero di Capograssi e di Pigliaru, nell'introduzione a A. Pigliaru, La lezione di Capograssi, cit., p. 18.

44 Così lo chiama S. Satta, Il giurista Capograssi (1960), in Id., Soliloqui e colloqui di un giurista, a cura di F. Mazzarella, Ilisso, Nuoro 2004, p. 366. 
dell'io a quello dell'azione umana, seguendo l'ispirazione di concetti capograssiani quali «esperienza comune» ed «esperienza giuridica» ${ }^{45}$. D'altro canto, sotto un profilo metodologico, l'attenzione alla socialità del diritto come esperienza giuridica dell'azione impone di studiare il fenomeno dell'ordinamento giuridico «dal basso $»^{46}$, ovverosia a partire dall'etica della comunità umana che diventa (e dunque è logicamente antecedente al) «diritto». A tal proposito, gli studiosi concordano sul fatto che la lezione capograssiana abbia offerto a Pigliaru l'occasione per «prendere in considerazione l'ordinamento di una comunità storica come era quella barbaricina», rivedendo «interamente i presupposti del proprio lavoro ${ }^{47}$. Sembra incontestabile, d'altra parte, la matrice capograssiana ${ }^{48}$ - oltre che marxiana e gramsciana - del postumo Studio sull'estinzione dello Stato (1975), dove si richiama espressamente il saggio su La nuova democrazia diretta $(1922)^{49}$.

È interessante, a questo punto, notare come, dall'interazione fra la precedente formazione dell'autore e la lezione capograssiana, le idee pigliariane sulla relazionalità umana si sviluppino ulteriormente secondo

45 È noto, infatti, come l'autore orunese abbia per questa ragione riveduto «l'evidente equivoco» che lo aveva portato, in passato, a criticare le dottrine pluraliste (A. Pigliaru, La vendetta barbaricina come ordinamento giuridico, in Id., Il banditismo in Sardegna, Giuffrè, Milano 1970, p. 42, nota 24), richiamandosi anche (ivi, p. 27) ad un passaggio di Capograssi, Il problema della scienza del diritto, Soc. ed. Il Foro Italiano, Roma 1937, p. 42, a mente del quale «è proprio l'azione che prende se stessa come oggetto che si reduplica, che si osserva e si cerca di tradurre in un sistema di ordini e di determinazioni».

${ }_{46}$ A. Delogu, L’insegnamento di Capograssi, cit., p. 13.
${ }_{47}$ P. Carta, Pensiero giuridico e riflessione politica in Antonio Pigliaru, cit., p. 355.
Così nota V. Atripaldi, La Regione come «via italiana all'estinzione dello Stato», in «Ichnusa», n.s. VIII (1989), 18/19, p. 41.

49 A. Pigliaru, Studio sull'estinzione dello Stato, in Id., Scritti di Scienza politica, cit., p. 335: «più il discorso sullo Stato viene approfondito dall'esperienza [...] più cresce il rilievo teorico e pratico di quel problema della sua estinzione, che anche la conclusione di un importante libro del 1922 indicava come il tema necessario di ogni seria meditazione sullo Stato». 
la linea di tendenza già mostrata in passato. «Se si accetta la cosiddetta teoria della socialità del diritto», afferma il nostro autore, si può definire l'ordinamento giuridico «come il momento, il prodotto culminante in tutto il complesso processo onde l'azione, prendendo se stessa per oggetto, pone e realizza attivamente quella serie di comandi e quindi di obbedienze che creano i rapporti per cui la vita associata è effettivamente tale ${ }^{50}$. Queste parole de La vendetta barbaricina si riallacciano alla teorica capograssiana che esalta l'aspetto "dinamico" dell'esperienza, in luogo di quello più "statico" dell'ordinamento, e postula un circolo virtuoso in cui si svolge lo sviluppo di azione ed ordine: «l'azione», infatti, «reclama e pone la necessità dell'ordine», la quale «a sua volta reclama e pone la necessità di ulteriori azioni dirette a soddisfare quella necessità dell'azione ${ }^{51}$. Così, il ritmo produttivo in cui le azioni individuali sono mediate dagli ordini, accentua la forza propulsiva e performante della relazione intersoggettiva, dal momento che lo sviluppo dell'azione attraverso il circolo azione-ordine presuppone l'interazione relazionale degli uomini. È significativo, d'altra parte, che l'evoluzione delle dottrine sull'esperienza giuridica, negli anni successivi, proceda proprio nella direzione dell'enfasi sull'attitudine relazionale del soggetto ${ }^{52}$.

Il momento del rapporto intersoggettivo si connette, peraltro, alla definizione stessa di «ordinamento giuridico», per la quale Pigliaru riprende le parole di Alessandro Levi (1881-1935) secondo cui l'ordinamento altro non sarebbe che «l'insieme organico dei rapporti giuridici in quanto sociali $\nu^{53}$. Nella prospettiva di quest'ultimo autore, infatti, il rapporto

50 A. Pigliaru, La vendetta barbaricina, cit., p. 22.

51 Capograssi, Il problema della scienza del diritto, cit., pp. 41-42.

52 Il nesso fra il concetto di esperienza e la dimensione dei rapporti intersoggettivi emerge, infatti, tanto nell'elaborazione storicistica di Riccardo Orestano (19091988) quanto nell'etica della «reciprocità» di Alessandro Giuliani (1925-1997), come emerge molto bene dalla ricostruzione di F. Cerrone, Sull'esperienza giuridica: Capograssi, Orestano, Giuliani, in «Diritto Pubblico», (2016), 3, pp. 963-1017.

53 A. Pigliaru, La vendetta barbaricina, cit., p. 22. 
«rappresent[a] il concetto fondamentale del diritto ${ }^{54}$ ed il fenomeno giuridico serve a dar luogo «ad una proporzione, ad un rapporto di misura, fra uomo e uomo» ${ }^{55}$. Nello specifico, il filosofo di Orune accede alla concezione leviana che qualifica l'ordinamento in termini, anzitutto, di «posizione» di rapporti, poiché individua lo statuto giuridico dei soggetti all'interno della relazione (stabilendo, ad esempio, chi sia proprietario di una cosa e chi, invece, sia estraneo a tale titolo); inoltre, l'ordinamento è anche il luogo della «organizzazione» dei rapporti, nel senso che le sue norme stabiliscono attraverso quali modalità un soggetto può agire e muoversi, in modo giuridicamente legittimo, nell'ambito di una relazione (sancendo, ad esempio, quali diritti abbia il proprietario) $)^{56}$.

L'approdo pigliariano a questa concezione relazionale del diritto appare, dunque, evidente anche nella sua dottrina pluralistica, espressa nel saggio sulla vendetta in Barbagia. In questo famoso scritto del 1959, l'autore rivede la propria teoria dell'ordinamento, recuperando, grazie alla "nuova" affiliazione capograssiana, le prospettazioni di Santi Romano (1875-1947) e Georges Gurvitch (1894-1965), nei cui confronti si era mostrato molto critico, alcuni anni prima. Così facendo, Pigliaru addiviene alla propria teoria sulla pluralità degli ordinamenti giuridici, che risulta dalla sintesi delle elaborazioni di vari autori con un particolare accento sull'elemento relazionale. Infatti, riferendosi al legame sociale che avvince i membri della comunità barbaricina, il filosofo sardo parla di «comunità del $\mathcal{N}_{0} i$ », richiamandone la definizione gurvitchiana quale «unità non decomponibile nella pluralità dei membri», che tuttavia non assume «atteggiamenti di opposizione» nei confronti di essi ${ }^{57}$. Il nostro autore, tuttavia, non si rifà in toto alla concezio-

54 Levi, Teoria generale del diritto (1950), Cedam, Padova 1967, p. 11.

55 Levi, Contributi ad una teoria filosofica dell'ordine giuridico, Formiggini, Genova 1914, p. 177.

56 Levi, Teoria generale, cit., p. 41.

57 G. Gurvitch, Sociology of law, New York 1942, trad. ita. a cura di S. Cotta, Sociologia del diritto, Edizioni di Comunità, Milano 1957, p. 248; cfr. A. Pigliaru, La vendetta barbaricina, cit., p. 265. 
ne del sociologo franco-russo, ma si limita ad attingere alla sua Sociologia del diritto, ancora una volta, secondo una prospettiva "manipolatoria" alla ricerca di un'elaborazione autorevole tramite cui rinforzare le proprie tesi. La categoria del «noi», come abbiamo visto, entra nel dizionario pigliariano molto tempo prima, attraverso una prima rielaborazione delle idee gentiliane: per Gentile, infatti, il noi è «comunità che ha vita nell'atto stesso dell'individuo ${ }^{58}$; individuo che, a sua volta, supera l'alterità trovando, nell'intimità del noi, la (ri)composizione della comunanza. Tale comunanza fa sì che nell'azione individuale si manifesti il patrimonio etico della comunità stessa, la quale è dunque presente in ciò che il singolo fa e patisce.

Orbene, alla luce di quanto in precedenza affermato, si nota facilmente come l'approccio relazionale al diritto risieda, in Pigliaru, nel riferimento alla dimensione del noi. Tale categoria, infatti, identifica una relazione in cui si assiste alla reciproca partecipazione spirituale tra «persone» in virtù di ciò che esse hanno in comune. Una concezione siffatta riflette, a ben guardare, un modello sociale di matrice aristotelica, per cui ogni uomo, grazie al sentimento dell'amicizia ( $\varphi$ ı $\lambda$ í $\alpha$ ), è capace di riconoscersi nell'altro e di stringere con esso un legame, in ragione della possibilità che gli uomini hanno di soddisfare bisogni reciproci ${ }^{59}$. Il legame che io stringo con l'altro, proprio grazie al coinvolgimento spirituale esso implica, fa scattare la duplice dimensione tönniesiana della «comprensione» e della «concordia» ${ }^{60}$. Da questa particolare conformazione del noi come relazione sociale, emerge anche il profilo normativo

58 G. Gentile, Genesi e struttura della società, cit., p. 19.

59 Cfr. Aristotele, Etica Nicomachea, a cura di M. Zanatta, Rizzoli, Milano 1998, Vol. II, VIII, 1055a, 22-23, p. 705. A proposito del "modello aristotelico", cfr. T. Greco, Diritto e legame sociale, Giappichelli, Torino 2012, pp. 17-21.

60 Cfr. F. Tönnies, Gemeinschaft und Gesellschaft, Fues's Verlag, Leipzig, 1887, tr. it. a cura di G. Giordano e R. Treves, Comunità e società, Edizioni di Comunità, Milano 1963, pp. 62-64, dove si riferisce alla comprensione un «modo di sentire comune e reciproco» ed alla concordia il ruolo di raggruppare e regolare una molteplicità di comprensioni, ossia di relazioni. 
di quello che Pigliaru chiama «situazione», espressione tesa ad individuare quel complesso di circostanze, fattuali o sociali, caratteristiche di un dato contesto e, per ciò stesso, sottese ad ogni specifico ordine sociale. È importante sottolineare come l'autore impieghi la categoria della situazione non soltanto quando si riferisce alla realtà barbaricina, ma anche quando racconta e riflette sul mondo del carcere, dove il noi identifica un certo tipo di relazione umana (quella fra detenuti, «uomini di uno stesso destino, giusti ed ingiusti») e, quindi, un particolare tipo di situazione ${ }^{61}$. L'aspetto che più ci interessa evidenziare, in questa sede, è tuttavia costituito dal rapporto logico che connette la relazione umana all'ordine giuridico: infatti, la situazione svolge - come si è detto - una funzione normativa e strutturale rispetto all'ordinamento giuridico, la cui impalcatura deve attagliarsi alle caratteristiche delle relazioni che si sviluppano in un dato contesto. Così, ad esempio, laddove si sviluppa la cultura barbaricina del noi pastori, in ragione della particolare conformazione del territorio, dell'isolamento e delle asperità naturali, la regolamentazione della vendetta «emerge come principio ordinatore (e quindi come comando) nell'esperienza e dell'esperienza ${ }^{62}$. In virtù di queste considerazioni, si comprende, per un verso, come la relazione umana (sociale) rappresenti per Pigliaru un prius logico e teorico rispetto all'ordinamento, che quindi, pur costituendola giuridicamente, deve ad essa la propria esistenza ${ }^{63}$, potendosi sostenere, per altro verso, che la rilevanza del dato contestuale e della sovrapposizione fra ordina-

61 A. Pigliaru, Meditazioni sul regime penitenziario italiano (1959), Il Maestrale, Nuoro 2009, p. 26. A tal proposito, è interessante notare come Salvatore Mannuzzu (La terra vuota. Il Codice della vendetta, ieri e oggi, postfazione a A. Pigliaru, Meditazioni, cit., p. 97) spenda alcune parole per farci notare la vicinanza di questo tema al noi pastori della Vendetta barbaricina.

62 A. Pigliaru, La pratica della vendetta come ordinamento giuridico, saggio pubblicato postumo in Id. Il banditismo in Sardegna, a cura di L.M. Lombardi Satriani, Il Maestrale, Nuoro 2000, p. 506.

63 In questo modo, Pigliaru si mostra contrario al cosiddetto «modello hobbesiano» di legame sociale, che ritiene il diritto un elemento «costitutivo della possibilità 
menti rappresentino una sorta di preludio degli sviluppi ulteriori sperimentati dal pluralismo giuridico nella seconda metà del Novecento ${ }^{64}$.

Dopo aver osservato la risonanza del pensiero di Capograssi nella produzione del filosofo sardo, che lo ha indotto a rivisitare le proprie posizioni sul neoidealismo ${ }^{65}$, dovrebbe apparire chiara anche l'evoluzione della riflessione pigliariana in rapporto all'uomo ed alla categoria del noi. Il debito di Pigliaru nei confronti del filosofo di Sulmona è sicuramente importante ed evidente già a partire dal rinnovato vocabolario - particolarmente "capograssiano" - che presentano gli scritti degli anni Cinquanta e Sessanta. Tuttavia, il nostro autore non manca di leggere anche questo suo grande maestro in modo singolare ${ }^{66}$, a conferma di quanto detto sopra a proposito della strategia interpretativa di Pigliaru, il cui pensiero non avrà modo di formarsi compiutamente e sentirà sempre il bisogno di appoggiarsi ad altre autorevoli elaborazioni. In questo caso, la tesi che queste pagine pigliariane ci mostrano sembra inserirsi in quella linea di progressiva focalizzazione dell'interesse sul ruolo dell'azione umana nel mondo e sul protagonismo del soggetto in quanto uomo. Si tratta di un tema che l'autore, come vedremo, mai abbandonerà.

stessa delle relazioni umane» (T. Greco, Relazioni giuridiche. Una difesa dell'orizzontalità del diritto, in «Teoria e Critica della regolazione sociale», (2014), 1, p. 13).

64 La dimensione della cosiddetta «interlegalità» $\mathrm{o}$ «internormatività», che vorrebbe alludere alla sovrapposizione di fonti del diritto indipendenti ma connesse, riceve particolare attenzione nell'opera di vari autori contemporanei, che sottolineano la matrice contestuale del diritto (cfr. B. De Sousa Santos, Droit: une carte de lecture déformé. Pour une conception postmoderne du droit, in «Droit et Societé», (1998), 10), oppure problematizzano il rapporto fra sistemi normativi sovrapposti (cfr. M. Delmas-Marty, Le flou du droit. Du code pénal aux droits de l'homme, PUF, Paris 1986, a cura di F.C. Palazzo, tr. it, di A. Bernardi, Dal codice penale ai diritti dell'uomo, Giuffrè, Milano 1992).

65 Cfr. Delogu, L'eredità di Capograssi nel pensiero di Antonio Pigliaru, introduzione a A. Pigliaru, Saggi capograssiani, Edizioni Spes, Roma 2010, p. 37.

66 Su questa linea si muove anche P. Carta, Democrazia e lotta per il diritto, cit., pp. XXVI-XXVIII. 


\section{Il pensiero materialista ed il primato dell'uomo}

Lintervento di Pigliaru sui temi legati al marxismo ed alla lotta di classe emerge come un tassello ulteriore che compone il pensiero complessivo del nostro autore. Questo aspetto della riflessione pigliariana arriva più tardi rispetto all'esperienza filosofico-giuridica e filosofico-morale, di cui abbiamo già dato conto. L'attenzione che il nostro autore presta a questioni concrete è, d'altronde, cifra caratteristica della sua opera filosofica e teorica. È interessante notare come, all'occorrere, sempre più frequente, di questioni legate alle istanze autonomistiche della Sardegna, alle manifestazioni partitiche, ai movimenti studenteschi ed alla riforma agraria, si registri un progressivo avvicinamento di Pigliaru ad alcuni autori variamente connessi al pensiero marxista o socialista. Confrontandosi con i loro testi, il filosofo di Orune sviluppa ulteriormente la propria riflessione sull'uomo, esaltando il primato dell'azione e della relazione, soprattutto nell'ottica di una riforma politica e giuridica che dia maggior peso alla libertà ed alla responsabilità dei cittadini ${ }^{67}$.

A partire dai primi anni Sessanta, Pigliaru intraprende un percorso di revisione e rivisitazione della propria opera, raccogliendo ed interpretando il pensiero di Antonio Gramsci (1891-1937). Sono gli studi sul banditismo a sperimentare, per primi, la nuova chiave di lettura dell'uo-

67 È opportuno, a questo proposito, rinviare ad altre ricerche sul rapporto fra Pigliaru e la "questione sarda", con particolare riferimento ai problemi dell'autonomia regionale e del dibattito sul "piano di rinascita", a cui l'autore prende parte a cavallo tra gli anni Cinquanta e Sessanta (si vedano, ad esempio, F. Francioni, Questione sarda e questione meridionale. Gli strumenti Analitici di Antonio Gramsci e Antonio Pigliaru, in Il soldino dell'Anima. Antonio Pigliaru interroga Antonio Gramsci, a cura di Comitato Archivio Antonio Pigliaru, CUEC, Cagliari 2010, pp. 91 e ss.; S. Tola, Gli anni di Ichnusa. La rivista di Antonio Pigliaru nella Sardegna della rinascita, ETS, Pisa 1994). Vale la pena di sottolineare, comunque, che anche le posizioni espresse da Pigliaru su questi temi si legano strettamente alla sua concezione umanistica e relazionale, senza la quale difficilmente si comprendono la sua riflessione politica sulla democrazia ed il suo rapporto con il pensiero di Gramsci. 
mo e della società attraverso la filosofia della prassi ${ }^{68}$, come si vede nella Intervista su Orgosolo (1963), saggio che reca una lunga premessa ed un importante rinvio ad affermazioni gramsciane ${ }^{69}$. Tuttavia, ben presto si verifica altresì il connubio tra la riflessione gramsciana e l'inflessione umanistica già presente in Pigliaru, il quale insiste sempre di più sulla rilevanza tematica dell'«uomo» e della «relazione». Durante il suo ultimo corso di lezioni, dedicato alla crisi delle Università ed ai moti del Sessantotto, il nostro autore riprende le considerazioni di Gramsci sull'«essenza umana». Per il filosofo di Ales - come per Pigliaru, d'altronde - l'uomo è soggetto che «non può essere concepito se non come uomo storicamente determinato, cioè che si è sviluppato e vive in certe condizioni, in un determinato complesso sociale o insieme di rapporti sociali» ${ }^{70}$. Lo studioso di Orune, d'altro canto, riprende e sviluppa in questi termini il concetto di relazione, già formulato come reciproca partecipazione dei soggetti strumentale alla progressiva formazione dell'essenza umana. Il fondamento di tale «reciprocità», per Pigliaru, risiede «nella ipotesi che è legittimo parlare di essenza umana solo nella misura in cui se ne può parlare postulandola come l'insieme delle relazioni sociali» ${ }^{71}$. In tal modo, il nostro autore riprende Gramsci non solo per esaltare l'importanza

68 Cfr. Carta, Pensiero giuridico e riflessione politica, cit., p. 361.

69 Questo scritto si basa su alcuni fatti di cronaca relativi all'omicidio di una coppia inglese (i coniugi Townley), per mano di un abitante di Orgosolo. La vicenda sembrava mettere in discussione i risultati della ricerca su La vendetta barbaricina, sicché portò all'intervista di Pigliaru, il quale rispose: «ho sempre sostenuto che la pratica della vendetta in Barbagia non spiega tutto il banditismo, in quanto ne costituisce solo una delle componenti», non escludendosi la possibilità che la comunità opti per un altro sistema etico "purché ciò avvenga sul piano di una scelta reale» (A. Pigliaru, Intervista sul problema del banditismo in Sardegna, in Id., Il banditismo in Sardegna. La vendetta barbaricina, cit., pp. 356-357).

70 Gramsci, Quaderni del carcere (1948-1951), III, a cura di V. Gerratana, Einaudi, Torino $1975, Q .15, \S 10$, p. 1765, poi raccolto nelle sue $\mathcal{N}$ ote sul Machiavelli.

${ }^{71}$ Lezione dell'11 novembre 1968, in A. Pigliaru, Il rispetto dell'uomo, a cura di L.M. Lombardi Satriani e M.A. Cattaneo, Iniziative Culturali, Sassari 1980, p. 58. 
dell'azione umana riformatrice nel «blocco storico» di struttura e soprastruttura $^{72}$, ma anche e soprattutto per sottolineare la funzione costitutiva della relazione: il blocco storico è infatti composto dai «rapporti sociali in cui gli uomini reali si muovono ed operano» ${ }^{73}$. Così, Pigliaru sviluppa ulteriormente la dimensione relazionale della «persona», insistendo ancor più sulla tesi per cui il soggetto matura la propria "essenza" grazie a questa reciproca contingenza che lo lega agli altri. Ora, infatti, il soggetto pigliariano rimuove definitivamente la "maschera" (perché di persona si parla) e diventa «uomo» a tutti gli effetti.

Negli stessi anni, il nostro autore si accosta altresì al pensiero di un altro importante filosofo legato al marxismo: Jean-Paul Sartre (19051980). Ad attirare l'attenzione di Pigliaru sono, in particolare, le posizioni espresse dall'intellettuale parigino sul pensiero materialista nel tentativo di correggere alcuni dogmi del «marxismo volgare» e di evidenziare il fondamento ed i criteri d'intelligibilità della dialettica storica, liberandola da condizionamenti ontologici o naturalistici ${ }^{74}$. Richiamandosi alle riflessioni sartriane, Pigliaru sviluppa ulteriormente il tema del rapporto fra la sfera etica e quella giuridica, già emerso a cavallo del 1960. Nel saggio sulla Vendetta barbaricina (1959), infatti, si sussume l'ordinamento giuridico della vendetta sotto l'etica del noi pastori, mentre in La piazza e lo Stato (1961) si sottolinea come lo Stato (e, dunque, l'ordinamento giuridico) rappresenti sempre la proiezione di un problema umano, non essendovi «nessun momento della problematica dello Stato (etica, giuridica, politica), che non tenda a risolversi nel problema dell'uomo, e quindi che possa essere risolto senza che nello stesso tem-

72 A. Pigliaru, Struttura, soprastruttura e lotta per il diritto, cit., p. 17, dove richiama Gramsci, Il materialismo storico e la filosofia di Benedetto Croce (1949), Einaudi, Torino 1966 p. 39.

73 Gramsci, Il materialismo, cit., p. 191.

74 J.-P. Sartre, Critique de la raison dialectique, Gallimard, Paris 1960, tr. it. di P. Caruso, Critica della ragione dialettica, I. Teoria degli insiemi pratici, Il Saggiatore, Milano 1990, p. 75. 
po si risponda al corrispondente interrogativo sull'uomo» ${ }^{75}$. Nel 1965 , addirittura, la prospettiva di riferimento non si riduce più a quella dello «Stato», ma si estende a quella della «storia». Pigliaru vede nell'uomo un protagonista attivo (e non, cioè, un soggetto passivo) dell'evoluzione storica, sicché l'azione umana si pone come «possibile e necessario insignorimento di essa ${ }^{76}$. In questo modo, il nostro autore evoca le parole con cui Sartre definisce l'uomo come progetto e futuro, categorie di importanza nevralgica, utili ad affermare «la specificità dell'atto umano, che attraversa l'ambito sociale pur conservando le determinazione, e che trasforma il mondo (sia pure) sulla base di condizioni date ${ }^{77}$. È interessante notare, anche in questa sede, l'accento marcato che Pigliaru pone sulla tendenziale sovrapposizione tra l'azione come «progetto e futuro» e la «vita di relazione» da cui essa scaturisce ${ }^{78}$. Il progetto spiega l'uomo, perché l'uomo «è per gli altri» ${ }^{79} \mathrm{e}$, dunque, la sua azione acquista senso in relazione agli altri. L’uomo, infatti, è tale in quanto «trascende» il proprio io e viene ad «esistenza fuori-di-sé in relazione con l'Altro-da-sé»" rimanendo dunque priva di "umanità" ogni esistenza che non contempli l'apertura all'altro, dal momento che, senza la relazione (e, dunque, senza la relazione umana), non può sussistere alcun «progetto».

\section{Conclusioni. La giustizia come carità in un inedito pigliariano}

Conviene, avviandoci alla conclusione, gettare uno sguardo d'insieme al tema pigliariano dell'umano, che si è cercato di analizzare attraverso un breve itinerario nella produzione dell'autore. Non stupisce, d'altra

75 A. Pigliaru, La piazza e lo Stato, cit., p. 48.

76 A. Pigliaru, Struttura, soprastruttura e lotta per il diritto, cit., p. 15, ma si veda anche Id., L'eredità di Gramsci e la cultura sarda, Il Maestrale, Nuoro 2008, p. 21.

77 Sartre, cit., p. 77.

78 A. Pigliaru, Struttura, soprastruttura e lotta per il diritto, cit., p. 15.

79 Ivi, p. 16, nota 9.

$80 \quad$ Sartre, cit., p. 131. 
parte, come l'attenzione rivolta dagli studiosi al problema umanistico in Pigliaru non abbia mai posto l'accento sull'elemento relazionale che ne caratterizza la riflessione. Infatti, il filosofo di Orune non ha mai discusso diffusamente e compiutamente la connessione fra uomo e relazione, né quella fra relazione umana e diritto, sicché la ricostruzione "postuma" di queste idee pigliariane, sulla base di una lettura trasversale dei suoi testi, potrebbe apparire meramente arbitraria. Tuttavia, non sembra esservi dubbio sulla rilevanza di queste tematiche in tutta l'opera filosofica dell'autore, al quale, evidentemente, stava molto a cuore il problema del soggetto, della persona, dell'uomo. Osservando con attenzione gli scritti pubblicati ed anche gli incompiuti, si può notare come queste tematiche emergano spesso, a volte in modo esplicito, altre volte tra le righe e le citazioni.

Un esempio interessante e suggestivo è dato da alcuni appunti annotati dall'autore per un progetto di scritto, mai realizzato, ora raccolti nel Quaderno 38 dell'Archivio «Antonio Pigliaru» ${ }^{81}$. Dalla lettura di queste pagine, è possibile inferire sulla loro plausibile destinazione, ossia un saggio dedicato al rapporto fra giustizia e carità, oppure alla giustizia come carità. Si tratta di un argomento fondamentale della teologia morale cristiana, al quale Pigliaru sembra volersi accostare per riflettere su questa particolare ed antica forma di giustizia ${ }^{82}$. La carità, nella prospettiva cristiana, ha a che fare con l'amore ed esige, com'è noto, «il compimento della legge» nel rapporto con il prossimo, con l'uomo altroda-me, ma al contempo vicino a me ${ }^{83}$. La giustizia-carità si apprezza,

81 L'archivio è stato curato da Paolo Carta e Alessandra Pigliaru, attraverso la collezione di appunti e bozze nella biblioteca privata di casa Pigliaru.

82 Negli appunti si trovano fonti di varia matrice: oltre a vari passaggi biblici, si registra un'ampia gamma di citazioni da testi dottrinali e letterari (come, ad esempio, Il diavolo e il buon Dio di Sartre).

83 Cfr A. Pigliaru, Quaderno 38 (di seguito, Q. 38), p. 28, dove cita Paolo, Roma$n i, 12,8$ : «Non siate debitori con nessuno di nulla, fuorché nell'amore scambievole, perché chi ama il prossimo, ha adempiuto la legge». 
in particolare, entro una dinamica relazionale in cui l'io si apre all'altro più debole, all'orfano, all'oppresso, al prigioniero, all'affamato, all'assetato e così via ${ }^{84}$. A tal proposito, si può ricordare - come peraltro è già stato fatto - l'esempio evangelico del buon Samaritano, il quale, sceso da cavallo, si fa vicino all'uomo in difficoltà. In questo caso, la giustizia si realizza nella relazione e tramite la relazione stessa, giacché «l'atto di abbassarsi è metaforicamente un'uscita da se stessi per rivolgersi all'altro ${ }^{85}$. Pigliaru, d'altronde, sembra assecondare la medesima intuizione, come si evince da una riflessione personale scritta a mano in mezzo agli appunti. Per lui, fare la carità significa «mettere tra parentesi il debito gerarchico, o comunque metterci sopra un momento intersoggettivo» ${ }^{86}$, in virtù del quale la logica segmentaria e divisiva del diritto-obbligo non funziona più, dovendosi ad essa sostituire la logica del dovere rispetto al bisogno.

Si tratta, per il vero, di una posizione che conferma alcune idee espresse dal nostro autore fin dai suoi primi scritti, consentendoci pertanto di concludere che, in Pigliaru, la teoria della relazione come realizzazione dell'essenza umana si accompagna all'assunzione della relazione come contenuto prescrittivo della giustizia-carità. Secondo questa prospettiva, non vi è realizzazione dell'essenza umana senza giustizia, poiché non vi è giustizia senza relazione. In questi appunto, infatti, l'autore annota una frase sulla nozione di «giustizia», affermando che essa si caratterizza per tre «proprietà», consistenti nel «tendere ad altri (alterità)», «rendere quanto è dovuto»e, infine, «rendere fino all'uguaglianza» ${ }^{87}$. Alla luce di quanto affermato nelle pagine addietro, il richiamo a questo passaggio sembra rafforzare ulteriormente le tesi esposte. La frase sopracitata, in-

84 Cfr. Ignazio d'Antiochia, Lettera agli Smirnesi, VI, 1, citato in Q. 38, p. 30.

85 T. Greco, La giustizia del Crocifisso, in «Endoxa. Prospettive sul presente», I (2016), 4, p. 41.

86 A. Pigliaru, Q. 38, p. 16.

87 Ivi, p. 64, dove Pigliaru riprende la voce «giustizia» da un «Dizionario di teologia morale» non meglio specificato. 
fatti, compendia le idee che - come abbiamo visto - hanno accompagnato Pigliaru dalla gioventù alla maturità, fino alla morte. In queste parole risiedono l'elemento relazionale della giustizia (e del diritto), il profilo del dovere, nonché quello relativo al contenuto del dovere: il rispetto dovuto ad ogni uomo in quanto uomo, incarnato dal riferimento all'eguaglianza (data la comune umanità, infatti, è innegabilmente dovuto il medesimo rispetto a me come all'altro). Naturalmente, queste affermazioni non possono che rappresentare delle mere ipotesi, dal momento che oggi disponiamo solo di appunti accompagnati da pochissime e brevi considerazioni a margine. Tuttavia, pare innegabile la possibilità di suffragare tali ipotesi tramite l'attenta lettura degli scritti più importanti di Pigliaru, che ci permettono di notare come la sua costituzione teorica del soggetto consideri massimamente i profili dell'umano, della sua naturale apertura alla relazione con l'altro e, infine, del dovere di rispetto nei confronti dell'altro. 